\title{
A novel conceptual framework for balance training in Parkinson's disease-study protocol for a randomised controlled trial
}

\author{
David Conradsson ${ }^{1,2^{*}}$, Niklas Löfgren ${ }^{1}$, Agneta Ståhle ${ }^{1,2}$, Maria Hagströmer ${ }^{1}$ and Erika Franzén ${ }^{1,2}$
}

\begin{abstract}
Background: There is increasing scientific knowledge about the interaction between physiological (musculoskeletal, neuromuscular, cognitive and sensory) systems and their influence on balance and walking impairments in Parkinson's disease. We have developed a new conceptual framework for balance training, emphasising specific components of balance control related to Parkinson's disease symptoms by using highly challenging, progressive and varying training conditions. The primary aim of this proposed randomised controlled trial will be to investigate the short-term and long-term effects of a 10-week balance training regime in elderly with Parkinson's disease.

Methods/Design: Eighty participants with mild to moderate idiopathic Parkinson's disease will be recruited and randomly allocated to an intervention group receiving balance training or a control group whose participants will continue to receive their usual care. The intervention will consist of a 10-week group training regime (1-hour training, three times per week), which will be led by two physiotherapists to ensure training progression and safety. The conceptual framework will be applied by addressing specific balance components (sensory integration, anticipatory postural adjustments, motor agility, stability limits) through varying training conditions and structured progression. Assessment will be conducted through a multi-dimensional battery of outcomes, prior to and immediately after the 10-week intervention, and at 9 and 15 months' follow-up after entering the study. Primary outcome measures will be balance performance (assessed using the Mini Balance Evaluation Systems Test), change in gait velocity $(\mathrm{m} / \mathrm{s})$ between single and dual task walking, and fear of falling (evaluated using the Fall Efficacy Scale International).
\end{abstract}

Discussion: This study has the potential to provide new insight and knowledge of the effects of specific, varied and challenging balance training on a wide health spectrum in elderly with PD. If found to be effective, this pragmatic approach with translation of theory into practice, can be implemented in existing outpatient care.

Trial registration: NCT01417598

\section{Background}

Parkinson's disease (PD) is a major worldwide health problem [1], with a reported incidence rate ranging from 11 to $19 / 100,000$ per year in the European population [2]. Balance and walking impairments are present even in the early stages of PD [3-6] and have been shown to

\footnotetext{
* Correspondence: David.Conradsson.1@ki.se

'Department of Neurobiology, Care Sciences and Society, Division of Physiotherapy, Karolinska Institute, 23100, SE-14183, Huddinge, Sweden ${ }^{2}$ Department of Physical Therapy, Karolinska University Hospital, Stockholm,
} Sweden

\section{Biomed Central}

(c) 2012 Conradsson et al.; licensee BioMed Central Ltd. This is an Open Access article distributed under the terms of the Creative Commons Attribution License (http://creativecommons.org/licenses/by/2.0), which permits unrestricted use, distribution, and reproduction in any medium, provided the original work is properly cited. be associated with restrictions in everyday activities [7] and reduced quality of life (QoL) [8].

Furthermore, elderly with PD have shown reduced levels of physical activity [9] and a nine times increased risk of injurious falls, compared with the healthy elderly [10]. As PD progresses, balance problems gradually increase and are generally non-responsive to or worsen with levodopa treatment [11-13]. Currently, there is a growing body of research that highlights the role of physical exercise as an essential part of managing the disease, with potential neuroprotective mechanisms $[14,15]$. The effects of balance training on balance and 
gait performance are promising for individuals with PD [16-18], although several questions remain unanswered, particularly regarding dose, intensity and duration, as well as regarding specific exercises to improve balance control in the different stages of the disease [17-21]. Additionally, there is a lack of knowledge of the longterm effects of training interventions on participation in everyday living, such as physical activity and QoL [16].

Balance control relies on the interaction of several physiological systems (the musculoskeletal, neuromuscular, cognitive and sensory systems) with environmental factors and the performed task [22]. Balance impairment or postural instability in individuals with $\mathrm{PD}$ is often associated with poor or absent reactive responses following external perturbations, such as performing a rapid step following a slip or trip [23]. However, as degeneration of the basal ganglia affects many physiological systems essential for balance control, balance disorders in PD cannot be addressed to one single function but, rather, are the result of impairments of multiple systems [24-26]. First of all, dysfunction of the basal ganglia influences sensory integration, i.e. the ability of the central nervous system to transform different modalities of sensory information (somatosensory, visual and vestibular) into a single reference frame, which is important for estimation of limb and body position in relation to the environment [27]. Particularly, individuals with PD have shown impaired proprioception [13,27], overestimation of the amplitude of movements [28] and over-reliance on vision for balance control [29]. Moreover, the central motor drive has shown to be impaired in PD [30], causing bradykinetic movements and poorly timed and scaled anticipatory postural adjustments (APAs), i.e. feedforward control to stabilise the posture before and during voluntary movements [31,32]. Furthermore, the PD-characteristic "stooped" posture, decreased joint range of motion, narrow foot stance and axial rigidity increase the risk of instability since these musculoskeletal constraints reduce the functional limits of stability, i.e. the area in which the body centre of mass (CoM) can be moved with maintained stability and without changing the base of support (BoS) [33,34]. Taken together, sensory-motor interaction is essential for balance control and consequently, as discussed by Konczak et al. [27], sensory problems may degrade movement responses and cause instability to individuals with PD.

The functions of the basal ganglia incorporate motor program selection and adaptation $[35,36]$, here referred to as motor agility, which involves maintenance of coordination between body parts, task-specific adjustments of movement and quick shifts from one task to the next. Deficient motor regulation in PD, manifested as poor inter-segmental coordination [37], problems adopting postural synergies [35,38] and delayed change of motor commands when shifting from one task to another [39], is believed to contribute to increased instability and freezing of gait during fall-related activities $[40,41]$. Another critical aspect of balance control in PD is the ability to divide attention and simultaneously process multiple tasks (motor or cognitive), i.e. multi-tasking. While performing a multi-task activity, individuals with PD compared with healthy subjects are more inclined to shift attention away from the balance task, which can lead to falls [24].

To be successful, all types of training require the application of basic training principles of (i) specificity, i.e. being specific to the targeted function; (ii) progressive overload, i.e. providing a challenging overload to the physiological system through a certain level of intensity and regularity; and (iii) varied practice, i.e. promoting variation between exercise conditions $[42,43]$. To be specific, balance training needs to target functions, or impairments, of balance control associated with PD symptoms. As previously mentioned, there is increasing scientific knowledge concerning the interaction between and impact of different physiological systems for balance disorders in PD [24-26]. Previous intervention studies targeting balance performance in $\mathrm{PD}$ have, however, mainly emphasised non-specific training or compensatory training $[17,44]$. Moreover, to stimulate relearning of physiological systems important for balance control, the challenge level (i.e. intensity of the training, the difficulty level of balance exercises and the total training dose) needs to be considered. Allen et al. [17] recently concluded, based on a meta-analysis of exercise interventions targeting balance performance in $\mathrm{PD}$, that previous studies in general have used low challenge levels, suggesting that future exercise interventions should focus on more challenging aspects of balance exercises and a higher training dose. Furthermore, to enhance generalisation of learned motor skills to a wide variety of situations and promote a multi-faceted repertoire of movement strategies, it is essential to guarantee practice through a wide variety of balance exercise conditions [43].

We hypothesise that a new conceptual framework for balance training, emphasising critical aspects of balance control specifically related to PD symptoms through highly challenging, progressive and varied exercises, will improve, or maintain, balance and gait performance in elderly with PD. Moreover, we predict that increased physical functioning following training will lead to greater confidence in everyday participation, improved QoL and increased levels of physical activity.

The primary aim of this proposed randomised controlled trial will be to expose elderly individuals with mild to moderate PD to a 10-week balance training regime, and compare the efficacy of balance training 
to care as usual using laboratory, clinical, free-living and self-perceived assessments before and immediately after the intervention as well as 9 and 15 months post-baseline.

\section{Methods \\ Design}

In a prospective, randomised controlled clinical trial, with 9 and 15 months' post-baseline follow-up, 80 elderly patients with PD living in Stockholm County, Sweden, will be included. The project has been approved by the Regional Board of Ethics in Stockholm (Dnr: 2009/819-32 and 2010/1472-32).

\section{Participant selection}

Community-dwelling individuals with PD will be recruited from Karolinska University Hospital, the Swedish Parkinson Associations and outpatient neurological clinics as well as by physicians with a specialty in neurology in Stockholm County. Advertisements in local newspapers will also be used to recruit participants. Potential participants will receive written information about the aims of the study and its procedure, with an invitation to indicate their interest in participating to the study coordinator. Inclusion criteria will be a clinical diagnosis of "idiopathic" PD, according to the Queens Square Brain Bank criteria, $\geq 60$ years of age, have adapted to their present PD-medication and the ability to independently ambulate indoors without a walking aid. We will include mild to moderate PD (Hoehn and Yahr scores II and III) [45], since physical group training has been proved feasible for this level of disease severity $[46,47]$. Exclusion criteria will be a history suggesting "atypical" PD symptoms, as defined by Hughes et al. [48], a Mini-Mental State Examination (MMSE) score $\leq 24$ [49] and other existing neuromuscular disorders or medical conditions that substantially influences their gait and balance performance or their participation in the training program. To screen potential participants' eligibility, a physiotherapist will conduct a telephone interview based on a clinical self-report form covering questions about personal and medical history and selfperceived balance performance.

\section{Randomisation}

Participants will be divided into two geographic cohorts (south and north) to reduce the travelling time for the participants. After baseline assessments, the participants in each cohort will be randomly assigned to two different groups in blocks of four, one experimental group with balance training and one control group. The sequence of allocation will be performed using web-based software and will be concealed to the participants and testers until the baseline measurement is completed.
Blinding of group allocation will, however, not be possible since the test leaders will be supervising the training intervention (as described below).

\section{Intervention group}

We have applied the training principles of specificity, varied practice and progressive overload to design a conceptual framework for balance training in PD. The framework comprises specific exercises associated with balance and walking constraints in PD (Table 1). The intervention period is divided into three blocks (A, B and $\mathrm{C}$ ) to promote appropriate progressive overload through the course of training (Table 2). Implementation of the training principles for balance training and motor learning is outlined below.

\section{Specificity}

In contrast to non-specific training in PD [17], King and Horak [25] recently proposed a symptom-specific training approach targeting particular mobility impairments in individuals with PD. Our approach towards balance training resembles theirs regarding the principle of specificity. In the proposed intervention, we will use four balance components (sensory integration, APAs, motor agility, and stability limits) to target symptom-specific balance impairments associated with instability and falls in PD (see Table 1). The applications of these balance components will be separated according to exercise principles and objectives.

\section{Varied practice}

Through the course of training, exercise variation will be emphasised to enhance motor learning and promote generalisation of balance skills to everyday activities [43]. Based on previous findings, individuals with PD, compared with healthy individuals, need more time to achieve motor learning [50,51]. Therefore, as illustrated in Table 2, variation in the training will be increased successively through the course of training. During the initial phase of training (Block A), the exercise for each balance component will be trained separately in blocks on a weekly basis, to encourage familiarity of the principles and objectives and promote task-specific motor learning. In the later phases of training (Blocks B and C), generalisation of motor skills will be facilitated by increasing training variation with regard to characteristics of exercise in each balance component (e.g. variation in terms of body position, BoS and movement direction/ velocity/amplitude) in Block $B$, and by integrating exercises from different balance components in Block $\mathrm{C}$. In addition, multi-task exercises in Blocks $B$ and $C$ will further increase training variation (see Table 2). 
Table 1 Parkinson's disease (PD) specific balance components, constraints affecting balance and exercises designed to reduce these constraints

\begin{tabular}{|c|c|c|c|}
\hline Balance components & Constraints in PD & Exercise principles & Exercise objectives \\
\hline \multicolumn{4}{|l|}{ SENSORY INTEGRATION } \\
\hline \multirow{3}{*}{$\begin{array}{l}\text { Integration of sensory information } \\
\text { (somatosensory, visual and vestibular) } \\
\text { for estimation of body position }\end{array}$} & - Impaired somatosensory integration & \multirow{3}{*}{$\begin{array}{l}\text { Walking tasks on varying surface } \\
\text { with or without visual constraints }\end{array}$} & \multirow{3}{*}{$\begin{array}{l}\text { Improve interpretation of and } \\
\text { reliance on somatosensory } \\
\text { information }\end{array}$} \\
\hline & - Poor proprioception & & \\
\hline & - Visual dependency & & \\
\hline
\end{tabular}

\section{APAs}

Prediction and control of perturbation - Poorly timed and scaled APAs

related to voluntary movements

Voluntary arm/leg/trunk movements focusing on movement velocity and amplitude, and postural transitions
Improve APA strategies regarding quality (timing, amplitude) and task- specific adaptation

\section{MOTOR AGILITY}

Coordination between body parts and movement adaptation, e.g. regulation of movement and quick shifts between tasks

\author{
- Bradykinesia \\ - Impaired whole-body coordination \\ - Biomechanical constraints \\ - Inflexible motor programming
}

Whole-body coordination during varying gait conditions and reciprocal movements. Quick shifts of movement characteristics (velocity, amplitude and direction) during predictable and unpredictable conditions
Improve whole-body coordination, ability to adapt movement and quick shifts between different tasks

\section{STABILITY LIMITS}

Whole-body regulation relative to - Reduced functional stability limits Voluntary leaning tasks in standing the BoS
- Biomechanical constraints

- Poor proprioception

- Impaired somatosensory integration with varying BoS-stimulating weight shifts in multiple directions through arm and trunk movements
Improve the ability to safely control CoM within BoS to increase functional limits of stability

APAs = anticipatory postural adjustments; $\mathrm{BoS}=$ base of support; $\mathrm{CoM}=$ centre of mass.

\section{Progressive overload}

Based on previous recommendations [17] and national guidelines for physical training [52], as well as our experience from a pilot study of balance training in PD (data not yet published) and balance training in elderly [53], we believe that the training frequency, duration and total training dose planned for use in the proposed study (three 1-hour sessions/week during 10 weeks) will be sufficient to improve balance performance in elderly with mild to moderate PD. To achieve optimised challenges to individual capacities, the physiotherapists will individually adjust the difficulty level of the exercises for each balance component as follows:

- Sensory integration: increased surface unevenness and restricted field of vision (vision-restricting glasses, carrying an object)

- APAs: increased movement amplitude and velocity

Table 2 Training progression

\begin{tabular}{|c|c|c|c|c|}
\hline & Training principles and objectives & Week & Balance components & Multi-task \\
\hline \multirow[t]{2}{*}{$\mathrm{A}$} & \multirow{2}{*}{$\begin{array}{l}\text { Introduction of performance of each balance component } \\
\text { separately and emphasizing quality of performance to } \\
\text { accomplish familiarity and task-specific motor learning. }\end{array}$} & 1 & Motor agility/stability limits & \\
\hline & & 2 & Sensory integration/APAs & \\
\hline \multirow[t]{4}{*}{ B } & \multirow{4}{*}{$\begin{array}{l}\text { Improvement of balance performance and strategies of } \\
\text { attention in varying balance conditions through increased } \\
\text { level of difficulty and task variation for each balance } \\
\text { component separately, and by using multi-tasking } \\
\text { (i.e. cognitive or motor secondary task). }\end{array}$} & 3 & Motor agility/stability limits & C-DT \\
\hline & & 4 & Sensory integration/APAs & M-DT \\
\hline & & 5 & Motor agility/stability limits & C-DT \\
\hline & & 6 & Sensory integration/APAs & M-DT \\
\hline \multirow[t]{4}{*}{$C$} & \multirow{4}{*}{$\begin{array}{l}\text { Further challenging of movement complexity through } \\
\text { increased levels of difficulty, task variation by successively } \\
\text { integrating the balance components, and increasing demands } \\
\text { of multi-tasking (i.e. cognitive and motor secondary tasks are } \\
\text { performed simultaneously). }\end{array}$} & 7 & Sensory integration/APAs/motor agility/stability limits & $C+M-D T$ \\
\hline & & 8 & Sensory integration/APAs/motor agility/stability limits & $C+M-D T$ \\
\hline & & 9 & Sensory integration/APAs/motor agility/stability limits & $C+M-D T$ \\
\hline & & 10 & Sensory integration/APAs/motor agility/stability limits & $C+M-D T$ \\
\hline
\end{tabular}

The balance program divided into three blocks (Blocks $\mathrm{A}-\mathrm{C}$ ), with training principles and objectives for each block.

APAs = anticipatory postural adjustments; C-DT = cognitive dual-task training; M-DT = motor dual-task training; $C+M-D T=$ mixed cognitive and motor dual-task training. 
- Motor agility: increased gait complexity (e.g. altering velocity, step patterns, etc.), increased gait demands according to planned (awareness of upcoming sequences/tasks) and unplanned shifts upon verbal commands (unawareness of upcoming sequences/ tasks) between different gait conditions, reciprocal movements and walking directions

- Stability limits: changing the area or condition of the BoS and increasing leaning movement amplitude

Intermittent presence of reactive postural adjustments will be used as an indicator of the appropriate difficulty level. To target multi-task performance in PD, cognitive (e.g. counting, remembering items/numbers) and secondary motor tasks (e.g. carrying and/or manipulating objects) [54], will be introduced in Block B and further progressed in Block C (see Table 2). Multi-task training will be individually adjusted for each participant. The level of difficulty for the secondary tasks (motor or cognitive) will be considered appropriate if the primary task is affected (e.g. decreased gait velocity or increased stride variation). Moreover, as described above, training demands will additionally be challenged by increasing variation through the course of the program.

\section{Feedback}

In individuals with PD, goal-oriented oral feedback that addresses information externally to the body, i.e. concerning the performance of the task in relation to the environment (rather than the position or motion of the body), has been reported to enhance walking [55] and balance performance [56]. In the proposed trial, oral feedback will be as simple as possible and be externally oriented to promote movement automaticity. Cueing strategies (visual or oral), as described by Nieuwboer et al. [57], will be used when needed for participants experiencing severe freezing through the course of training; however, they will not be used as a standard training component.

\section{Training/intervention procedure}

The balance training will be performed in groups of five to seven participants in the facilities of the Physiotherapy Clinic at Karolinska University Hospital. To secure progression and safety, all training sessions will be led by two physiotherapists with experience of working with PD and elderly patients. To standardise the intervention and secure adherence to the proposed theoretical framework, all physiotherapists will participate in a theoretical and practical workshop before the intervention starts and will be supervised during the course of the training.

Every training session will start with a warm-up session for $5 \mathrm{~min}$, consisting of varied walking tasks aiming to boost the cardiovascular system. The following $50 \mathrm{~min}$ (short rests included) will focus on highly challenging exercise blocks (approximately 10 min per block) of standing and walking conditions. The balance components of stability limits and APAs will mainly be addressed through standing exercises, and the balance components of motor agility and sensory integration will mainly be addressed in walking exercise conditions.

The program will end with a 5-min cool-down session with slow walking, axial stretching and breathing exercises. The number of exercise blocks for each training occasion and the distribution between standing and walking exercises will be adjusted to the level of ability in each group. After each training occasion, individuals' performance of the exercises included will be briefly documented, as an evaluation of both the appropriateness of exercises and the current level of difficulty. Adverse events (defined as an injury or medical event that restricts everyday activities and participation in the intervention) and training adherence will be monitored and recorded [58].

\section{Exercise on prescription}

We predict that increased physical functioning following training will lead to greater confidence in everyday participation and therefore an increased level of physical activity. However, targeted advice is needed for longterm behavioural change $[59,60]$. Therefore, a meeting will take place after the last training session, where the participants will participate in a group discussion, led by physiotherapists, on how to sustain or increase their level of physical activity. Participants will be given Physical Activity on Prescription (PaP) [59,60], together with a leaflet on benefits of physical activity and health in PD.

\section{Control group}

The subjects in the control group will receive usual care from their medical practitioner and/or the community services, and will not be restricted in participating in ongoing rehabilitation programs. The control group will be offered the same balance program after study termination.

\section{Outcome measures and test procedure}

According to the wide disability spectrum associated with balance impairments in PD [16,61], a multidimensional battery of outcomes will be used to identify potential training effects. As illustrated in Table 3, we have applied the International Classification of Functioning, Disability and Health (ICF) as a conceptual framework to target relevant constructs of body functions (i.e. physiological functions of body systems), activity (i.e. execution of a task or action by an individual) and participation (i.e. involvement in a life situation) [62]. As previously suggested by Dibble et al. [16], our outcome 
Table 3 Classification of outcome measures using the International Classification of Functioning, Disability and Health (ICF)

\begin{tabular}{|c|c|c|c|c|}
\hline \multirow[b]{2}{*}{ Instruments } & \multirow[b]{2}{*}{ Domains } & \multicolumn{3}{|c|}{ ICF } \\
\hline & & Body functions & Activity & Participation \\
\hline \multicolumn{5}{|l|}{ Laboratory tests } \\
\hline - Electronic walkway ${ }^{*}$ & Temporal and spatial gait parameters during single/multi-tasking conditions & $x$ & $x$ & \\
\hline - Mod-CTSIB & Sensory integration & $x$ & & \\
\hline \multicolumn{5}{|l|}{ Clinical tests } \\
\hline - Mini-BESTest & Subdomains of balance performance & $x$ & $x$ & \\
\hline - One-leg stance test & Balance performance, standing on one leg & & $x$ & \\
\hline - Modified figure of eight test & Balance performance, walking in a figure of eight with reduced BoS & & $x$ & \\
\hline \multicolumn{5}{|l|}{ Free-living assessment } \\
\hline - Accelerometer & Physical activity & & $x$ & \\
\hline \multicolumn{5}{|l|}{ Questionnaires } \\
\hline - FES-I & Fear of falling & & $x$ & \\
\hline - UPDRS ADL & Activities of daily living & & $x$ & \\
\hline$-\mathrm{SF}-36$ & Generic health-related QoL & & & $x$ \\
\hline - PDQ-39 & PD-specific health-related QoL & & & $x$ \\
\hline
\end{tabular}

"GAITRite, CIR Systems Inc., Clifton, NJ, USA.

BoS = base of support; PD = Parkinson's disease; QoL = quality of life.

FES-I = Fall Efficacy Scale International; Mini-BESTest = Mini Balance Evaluation Systems Test; Mod-CTSIB= Modified Clinical Test of Sensory Interaction and Balance; PDQ-39 = Parkinson's Disease Questionnaire; SF-36=36-item Short-Form Health Survey; UPDRS ADL = the activities of daily living (ADL) section of the Unified. Parkinson's Disease Rating Scale, part II.

measures represent a wide spectrum within the concepts of disability and health, from laboratory measurement of balance and gait performance, to free-living assessment of physical activity and self-perceived domains of QoL and participation in everyday living (see Table 3). Primary outcome measures will be functional balance performance, change in gait velocity $(\mathrm{m} / \mathrm{s})$ between single and dual task walking, and fear of falling.

Participants in both the intervention and the control group will be tested prior to and after the 10-week intervention, and at 9 and 15 months' follow-up after entering the study. All physical measurements will be conducted during the ON phase of their levodopa medication and at the same time of day to restrict the influence of medication fluctuations. The order of the physical tests (laboratory and clinical, as described below) will be randomised for each participant to avoid systematic bias.

Laboratory tests Temporal and spatial gait parameters will be measured using a $9 \mathrm{~m}$ electronic walkway (GAITRite, CIR Systems Inc., Clifton, NJ, USA) sensing the geometry of each step through embedded pressure sensors. Four types of gait conditions will be evaluated during (i) self-selected and (ii) fast speed, (iii) while performing a cognitive (reciting every second letter of the alphabet) and (iv) a motor dual-task (carrying a tray with a glass of water). Spatial and temporal gait parameters will be analysed, as will variability and asymmetry in gait performance.
The Modified Clinical Test of Sensory Interaction and Balance (Mod-CTSIB) will be used to measure sensory integration during static standing [63]. To discriminate between the sensory systems (somatosensory, visual and vestibular), participants will be timed while standing on a force plate $(50 \mathrm{~cm} \times 50 \mathrm{~cm}$; Kistler, Winterthur, Switzerland) in four different sensory conditions: (i) standing on a firm surface with the eyes open; (ii) standing on a firm surface with the eyes closed; (iii) standing on a compliant surface with the eyes open; and (iv) standing on a compliant surface with the eyes closed. Outcomes will be the time able to stand ( $\max 30 \mathrm{~s}$ ) and postural sway in the anterior-posterior and medio-lateral direction.

Clinical tests The Mini Balance Evaluation Systems Test (Mini-BESTest) will be used to assess underlying subsystems of balance performance (APAs, postural reactive responses, sensory integration and dynamic gait) [64].

The One-leg stance test will be performed with the subject standing on one leg as long as they are able to [65]. This test requires appropriate APAs for CoM transition from a standing position to a restricted BoS.

The Modified figure of eight test will be used to evaluate dynamic balance during walking [66]. The subject is instructed to walk in a figure of eight (marked with $40 \mathrm{~mm}$-wide tape on the floor, each loop having an internal diameter of $1.63 \mathrm{~m}$ ) as fast as possible, with every step touching the tape [66]. 
Free-living assessment Physical activity in everyday life will be assessed with accelerometers (Actigraph GT3X+, Manufacturing Technology Inc., Fort Walton Beach, FL, USA). The accelerometer will be used under free-living conditions, worn around the waist, using an elasticized belt, for 7 consecutive days. Outcomes will be total physical activity, expressed as counts per minute and steps per day, and time spent on different intensity levels as well as time spent sedentary [67].

Questionnaires The Activities of Daily Living section of the Unified Parkinson's Disease Rating Scale (UPDRS $A D L)$, part II, will be used to measure activities of daily living [68].

The Falls Efficacy Scale International-I (FES-I) will be used to measure participants concern of fear of falling during a variety everyday activities (e.g. getting dressed, taking a shower, climbing stairs or going to the shop) [69].

As previously suggested by Brown et al. (2009), assessment of Health-related QoL in PD should include both a generic and a PD-specific instrument [70]. The Short Form-36 (SF-36) will be used to evaluate generic healthrelated QoL [70,71] and the 39-item Parkinson's Disease Questionnaire (PDQ-39) will be used to evaluate PDspecific health-related QoL [70,72].

\section{Statistical analysis}

Descriptive statistics will be performed to ensure comparability between scores at baseline as well as to test the assumption of homogeneity of variance for use of parametric statistics. To evaluate the effect of the intervention, mixed models (SPSS Inc., Chicago, IL, USA) with an alpha level of 0.05 will be used where appropriate. Significance of main or interaction effects will be explored using the Bonferroni post hoc test. In case of skewed distributed data, logarithmic transformations or corresponding non-parametric statistics will be used to assess main effects of the intervention.

\section{Sample size}

The sample size for this study was determined from a pilot study (not yet published), including five mild to moderate PD subjects who participated in the proposed balance training, and also from previous trials on patients with PD and elderly people $[53,73,74]$. For each sample size calculation, power was set at $80 \%$ and a two-sided test at the alpha level of $5 \%$. For the MiniBESTest, a sample size of 24 was required to detect a 3point difference between groups, assuming a mean variance or standard deviation (SD) of 3.6 (effect size: 0.83). For the interference effect of dual task performance, i.e. percent change in gait velocity $(\mathrm{m} / \mathrm{s})$ between single and dual task walking [74], a sample size of 27 subjects was required to detect a difference of $10 \%$ (SD 13) between groups (effect size $=0.77$ ). Finally, for the FES- I, a sample size of 32 subjects was required to detect a difference of 2 points (SD 2.8) between groups (effect size $=0.71$ ). The dropout rate was set at $15 \%$, corresponding to intervention studies with PD subjects $[75,76]$. Taken together, to ensure adequate statistical power the sample size for this study would optimally be 40 per group (total $\mathrm{n}=80$ ).

\section{Discussion}

In this study protocol, a conceptual framework based on current scientific knowledge from neuroscience constraints in PD is used to design a training intervention targeting balance and walking performance in PD. The rationale for this training approach relies on findings of preserved motor learning in mild to moderate stages of PD [51] and the plausibility of training-induced neuroprotection $[14,15]$. Although previous exercise interventions in PD have reported promising results for balance and gait performance [16-18], they often lack to report the underlying principles of the intervention [21]. In particular, knowledge concerning the influence of active elements of the intervention (e.g. exercise components, training dosage and intensity) is limited [21]. In contrast to the majority of previous training regimes that emphasise general physical training [17] or target single physiological systems (e.g. muscular strength, reactive postural adjustments), the active elements in this intervention cover traditional training principles of specificity, progressive overload and random training. Hence, these principles are modified to promote motor learning in PD through specific balance components related to PD symptoms during highly challenging and varying exercise conditions. There is an urgent need to investigate both short-term and long-term effects of new exercise strategies targeting balance control in PD [17].

Balance impairment in PD comprises a wide spectrum of disability and health [16]. Detailed long-term followup, 1 year post-intervention, in the proposed study will give a unique opportunity to address important questions concerning potential effects of and limitations for group-based balance training. First of all, the proposed study will be one of the first trials to provide knowledge of potential effects of exercise intervention according to the whole spectra of ICF domains, including laboratory measurements, clinical tests, and free-living and selfperceived assessments (see Table 3). Such knowledge will be essential for designing and improving exercise interventions in the future. Secondly, long-term follow-up will provide knowledge of the maintenance of potential training effects. This is particularly important for PD given the progressive deterioration of the disease in 
which regular periods of supervised balance training may be required to sustain physical performance.

Since this intervention will not be restricted to a fixed program with specific exercises, several steps will need to be taken to standardise the intervention by educating physiotherapists in the framework and implementation of the training approach, as well as supervising the physiotherapists during the course of training and monitoring training performance as well as progression. During the course of training, each training session is planned with regard to the different phases of the intervention (see Table 2) and adapted by the physiotherapist to meet individual capacities of the participants. The physiotherapists conducting the training will be responsible for transferring the conceptual framework into practice, using a pragmatic approach rather than restricting the intervention to a standardised protocol. Thus, translation of this research intervention into the clinical setting will be enhanced.

There are several limitations to the proposed study. The result of this intervention can only be generalised to individuals with mild to moderate PD [45] and group training in outpatient care. All measurements will be conducted at the same time of the day during the $\mathrm{ON}$ phase of levodopa medication. The total dose of medication will be monitored in a longitudinal perspective [77], though separating the potential effects of balance training from those of levodopa medication will be limited. Moreover, blinding of the test leaders will not be possible since they also will serve as support for the physiotherapists conducting the intervention. However, this proposed randomised controlled trial has the potential to provide new knowledge and a change in focus concerning balance interventions in PD.

\section{Competing interests}

The authors declare that they have no competing interests.

\section{Authors' contributions}

AS and EF contributed to the research design and obtained funding for the study. DC, EF and NL provided the design of the study, intervention and outcome measures. $\mathrm{MH}$ contributed with design of the Physical Activity on Prescription-part of the intervention. DC was principally responsible for the drafting of the manuscript. All authors critically reviewed the manuscript and approved the submitted version.

\footnotetext{
Acknowledgement

The authors acknowledge Professor Johan Lökk for valuable discussions regarding assessment of Parkinson's disease medication. This study proposal is supported by grants from the Swedish Research Council, Karolinska Institute, the Loo and Hans Ostermans Foundation, the Gun and Bertil Stohnes Foundation, the Swedish Association of Persons with Neurological Disabilities (NHR), the Parkinson Foundation in Sweden and the regional agreement on medical training and clinical research (ALF) between Stockholm County Council and Karolinska Institute.
}

Received: 31 May 2012 Accepted: 25 September 2012 Published: 27 September 2012

\section{References}

1. Dorsey ER, Constantinescu R, Thompson JP, Biglan KM, Holloway RG, Kieburtz K, Marshall FJ, Ravina BM, Schifitto G, Siderowf A, Tanner CM: Projected number of people with Parkinson disease in the most populous nations, 2005 through 2030. Neurol 2007, 68:384-386.

2. de Rijk MC, Tzourio C, Breteler MM, Dartigues JF, Amaducci L, Lopez-Pousa S, Manubens-Bertran JM, Alperovitch A, Rocca WA: Prevalence of parkinsonism and Parkinson's disease in Europe: the EUROPARKINSON Collaborative Study. European Community Concerted Action on the Epidemiology of Parkinson's disease. J Neurol Neurosurg Psychiatry 1997, 62:10-15.

3. Carpinella I, Crenna P, Calabrese E, Rabuffetti M, Mazzoleni P, Nemni R, Ferrarin M: Locomotor function in the early stage of Parkinson's disease. IEEE Trans Neural Syst Rehabil Eng 2007, 15:543-551.

4. Galna B, Murphy AT, Morris ME: Obstacle crossing in people with Parkinson's disease: foot clearance and spatiotemporal deficits. Hum Mov Sci 2010, 29:843-852.

5. Beuter A, Hernandez R, Rigal R, Modolo J, Blanchet PJ: Postural sway and effect of levodopa in early Parkinson's disease. Can J Neurol Sci 2008, 35:65-68.

6. Suarez H, Geisinger D, Suarez A, Carrera X, Buzo R, Amorin I: Postural control and sensory perception in patients with Parkinson's disease. Acta Otolaryngol 2009, 129:354-360.

7. Tan DM, McGinley JL, Danoudis ME, lansek R, Morris ME: Freezing of gait and activity limitations in people with Parkinson's disease. Arch Phys Med Rehabil 2011, 92:1159-1165.

8. Ellis T, Cavanaugh JT, Earhart GM, Ford MP, Foreman KB, Dibble LE: Which measures of physical function and motor impairment best predict quality of life in Parkinson's disease? Parkinsonism Relat Disord 2011, 17:693-7.

9. Fertl E, Doppelbauer A, Auff E: Physical activity and sports in patients suffering from Parkinson's disease in comparison with healthy seniors. J Neural Transm Park Dis Dement Sect 1993, 5:157-161.

10. Bloem BR, Grimbergen YA, Cramer M, Willemsen M, Zwinderman AH: Prospective assessment of falls in Parkinson's disease. J Neurol 2001, 248:950-958.

11. Franzen E, Paquette C, Gurfinkel VS, Cordo PJ, Nutt JG, Horak FB: Reduced performance in balance, walking and turning tasks is associated with increased neck tone in Parkinson's disease. Exp Neurol 2009, 219:430-438.

12. Horak FB, Frank J, Nutt J: Effects of dopamine on postural control in parkinsonian subjects: scaling, set, and tone. J Neurophysiol 1996, 75:2380-2396.

13. Wright WG, Gurfinkel VS, King LA, Nutt JG, Cordo PJ, Horak FB: Axial kinesthesia is impaired in Parkinson's disease: effects of levodopa. Exp Neurol 2010, 225:202-209.

14. Ahlskog JE: Does vigorous exercise have a neuroprotective effect in Parkinson disease? Neurol 2011, 77:288-294

15. Hirsch MA, Farley BG: Exercise and neuroplasticity in persons living with Parkinson's disease. Eur J Phys Rehabil Med 2009, 45:215-229.

16. Dibble LE, Addison O, Papa E: The effects of exercise on balance in persons with Parkinson's disease: a systematic review across the disability spectrum. J Neurol Phys Ther 2009, 33:14-26.

17. Allen NE, Sherrington C, Paul SS, Canning CG: Balance and falls in Parkinson's disease: a meta-analysis of the effect of exercise and motor training. Mov Disord 2011, 26:1605-1615.

18. Crizzle AM, Newhouse IJ: Is physical exercise beneficial for persons with Parkinson's disease? Clin J Sport Med 2006, 16:422-425.

19. Keus SH, Bloem BR, Hendriks EJ, Bredero-Cohen AB, Munneke M: Evidencebased analysis of physical therapy in Parkinson's disease with recommendations for practice and research. Mov Disord 2007, 22:451-460.

20. Kwakkel G, de Goede CJ, van Wegen EE: Impact of physical therapy for Parkinson's disease: a critical review of the literature. Parkinsonism Relat Disord 2007, 13(Suppl 3):478-487.

21. Goodwin VA, Richards SH, Taylor RS, Taylor AH, Campbell JL: The effectiveness of exercise interventions for people with Parkinson's disease: a systematic review and meta-analysis. Mov Disord 2008, 23:631-640.

22. Horak F, Macpherson JM: Postural orientation and equilibrium. In Handbook of Physiology: Integration of Motor, Circulatory, Respiratory and Metabolic Control During Exercise. Edited by Rowell LB, Shepard JT. New York: Oxford University Press; 1996:255-92. 
23. Horak FB, Dimitrova D, Nutt JG: Direction-specific postural instability in subjects with Parkinson's disease. Exp Neurol 2005, 193:504-521.

24. Bloem BR, Grimbergen YA, van Dijk JG, Munneke M: The "posture second" strategy: a review of wrong priorities in Parkinson's disease. J Neurol Sci 2006, 248:196-204.

25. King LA, Horak FB: Delaying mobility disability in people with Parkinson disease using a sensorimotor agility exercise program. Phys Ther 2009, 89:384-393.

26. Bohnen NI, Cham R: Postural control, gait, and dopamine functions in parkinsonian movement disorders. Clin Geriatr Med 2006, 22:797-812.

27. Konczak J, Corcos DM, Horak F, Poizner H, Shapiro M, Tuite P, Volkmann J, Maschke M: Proprioception and motor control in Parkinson's disease. J Mot Behav 2009, 41:543-552.

28. Wright WG, Gurfinkel V, King L, Horak F: Parkinson's disease shows perceptuomotor asymmetry unrelated to motor symptoms. Neurosci Lett 2007, 417:10-15.

29. Paquette C, Franzen E, Jones GM, Horak FB: Walking in circles: navigation deficits from Parkinson's disease but not from cerebellar ataxia. Neuroscience 2011, 190:177-183.

30. Salenius S, Avikainen S, Kaakkola S, Hari R, Brown P: Defective cortical drive to muscle in Parkinson's disease and its improvement with levodopa. Brain 2002, 125:491-500.

31. Frank JS, Horak FB, Nutt J: Centrally initiated postural adjustments in parkinsonian patients on and off levodopa. J Neurophysiol 2000, 84:2440-2448.

32. Rocchi L, Chiari L, Mancini M, Carlson-Kuhta P, Gross A, Horak FB: Step initiation in Parkinson's disease: influence of initial stance conditions. Neurosci Lett 2006, 406:128-132.

33. Schenkman M, Morey M, Kuchibhatla M: Spinal flexibility and balance control among community-dwelling adults with and without Parkinson's disease. J Gerontol A Biol Sci Med Sci 2000, 55:441-445.

34. Mancini M, Rocchi L, Horak FB, Chiari L: Effects of Parkinson's disease and levodopa on functional limits of stability. Clin Biomech (Bristol, Avon) 2008, 23:450-458.

35. Chong RK, Horak FB, Woollacott MH: Parkinson's disease impairs the ability to change set quickly. J Neurol Sci 2000, 175:57-70.

36. Yehene $\mathrm{E}$, Meiran $\mathrm{N}$, Soroker $\mathrm{N}$ : Basal ganglia play a unique role in task switching within the frontal-subcortical circuits: evidence from patients with focal lesions. J Cogn Neurosci 2008, 20:1079-1093.

37. Bertram CP, Lemay M, Stelmach GE: The effect of Parkinson's disease on the control of multi-segmental coordination. Brain Cogn 2005, 57:16-20.

38. Chong RK, Jones $C L$, Horak FB: Postural set for balance control is normal in Alzheimer's but not in Parkinson's disease. J Gerontol A Biol Sci Med Sci 1999, 54:129-135.

39. Mak MK, Patla A, Hui-Chan C: Sudden turn during walking is impaired in people with Parkinson's disease. Exp Brain Res 2008, 190:43-51.

40. Spildooren J, Vercruysse S, Desloovere K, Vandenberghe W, Kerckhofs E, Nieuwboer A: Freezing of gait in Parkinson's disease: the impact of dualtasking and turning. Mov Disord 2010, 25:2563-2570

41. Ashburn A, Stack E, Ballinger C, Fazakarley L, Fitton C: The circumstances of falls among people with Parkinson's disease and the use of Falls Diaries to facilitate reporting. Disabil Rehabil 2008, 30:1205-1212.

42. Kraemer W: Musculoskeletal Exercise Prescription. In ACSM's Reasource Manual for Guidlines for Exercise Testing and Prescription. 6th edition. Edited by dA Ehrman J, Sanderson B, Swain D, Swank A, Womack C. London: Lippincot Williams \& Wilkins; 2010:463-475

43. Stergiou N, Harbourne R, Cavanaugh J: Optimal movement variability: a new theoretical perspective for neurologic physical therapy. J Neurol Phys Ther 2006, 30:120-129.

44. Morris ME, Martin CL, Schenkman ML: Striding out with Parkinson disease: evidence-based physical therapy for gait disorders. Phys Ther 2010, 90:280-288.

45. Hoehn MM, Yahr MD: Parkinsonism: onset, progression and mortality. Neurol 1967, 17:427-442.

46. Rodrigues de Paula F, Teixeira-Salmela LF, de Morais Faria CD C, Rocha de Brito P, Cardoso F: Impact of an exercise program on physical, emotional, and social aspects of quality of life of individuals with Parkinson's disease. Mov Disord 2006, 21:1073-1077.

47. States RA, Spierer DK, Salem Y: Long-term group exercise for people with Parkinson's disease: a feasibility study. J Neurol Phys Ther 2011, 35:122-128.
48. Hughes AJ, Daniel SE, Kilford L, Lees AJ: Accuracy of clinical diagnosis of idiopathic Parkinson's disease: a clinico-pathological study of 100 cases. J Neurol Neurosurg Psychiatry 1992, 55:181-184.

49. Folstein MF, Folstein SE, McHugh PR: "Mini-mental state". A practical method for grading the cognitive state of patients for the clinician. J Psychiatr Res 1975, 12:189-198.

50. Nieuwboer A, Rochester L, Muncks L, Swinnen SP: Motor learning in Parkinson's disease: limitations and potential for rehabilitation. Parkinsonism Relat Disord 2009, 15(Suppl 3):53-58.

51. Abbruzzese G, Trompetto C, Marinelli L: The rationale for motor learning in Parkinson's disease. Eur J Phys Rehabil Med 2009, 45:209-214.

52. Ståhle A: Physical activity in the prevention and treatment of diseases. Stockholm: Swedish National Institutet of Public Health; 2010.

53. Halvarsson A, Olsson E, Faren E, Pettersson A, Ståhle A: Effects of new, individually adjusted, progressive balance group training for elderly people with fear of falling and tend to fall: a randomized controlled trial. Clin Rehabil 2011, 25:1021-1031.

54. Brauer SG, Woollacott MH, Lamont R, Clewett S, O'Sullivan J, Silburn P, Mellick GD, Morris ME: Single and dual task gait training in people with Parkinson's disease: a protocol for a randomised controlled trial. $B M C$ Neurol 2011, 11:90.

55. Lebold CA, Almeida QJ: An evaluation of mechanisms underlying the influence of step cues on gait in Parkinson's disease. J Clin Neurosci 2011, 18:798-802.

56. Wulf $\mathrm{G}$, Landers $\mathrm{M}$, Lewthwaite $\mathrm{R}$, Tollner $\mathrm{T}$ : External focus instructions reduce postural instability in individuals with Parkinson disease. Phys Ther 2009, 89:162-168.

57. Nieuwboer A, Kwakkel $G$, Rochester $L$, Jones $D$, van Wegen $E$, Willems AM, Chavret F, Hetherington V, Baker K, Lim I: Cueing training in the home improves gait-related mobility in Parkinson's disease: the RESCUE trial. J Neurol Neurosurg Psychiatry 2007, 78:134-140.

58. Allen NE, Sherrington C, Suriyarachchi GD, Paul SS, Song J, Canning CG: Exercise and motor training in people with Parkinson's disease: a systematic review of participant characteristics, intervention delivery, retention rates, adherence, and adverse events in clinical trials. Parkinsons Dis 2012, 2012:854328.

59. Kallings LV, Leijon M, Hellenius ML, Stahle A: Physical activity on prescription in primary health care: a follow-up of physical activity level and quality of life. Scand J Med Sci Sports 2008, 18:154-161.

60. Leijon ME, Bendtsen P, Nilsen P, Festin K, Stahle A: Does a physical activity referral scheme improve the physical activity among routine primary health care patients? Scand J Med Sci Sports 2009, 19:627-636.

61. Morris ME: Locomotor training in people with Parkinson disease. Phys Ther 2006, 86:1426-1435.

62. Word Health Organization: International Classification of Functioning, Disability and Health. Geneva: ICF; 2001.

63. Cohen H, Blatchly CA, Gombash LL: A study of the clinical test of sensory interaction and balance. Phys Ther 1993, 73:346-351.

64. Franchignoni F, Horak F, Godi M, Nardone A, Giordano A: Using psychometric techniques to improve the Balance Evaluation Systems Test: the mini-BESTest. J Rehabil Med 2010, 42:323-331.

65. Jacobs JV, Horak FB, Tran VK, Nutt JG: Multiple balance tests improve the assessment of postural stability in subjects with Parkinson's disease. J Neurol Neurosurg Psychiatry 2006, 77:322-326.

66. Jarnlo GB NE: Reliability of the modified figure of eight - a balance performance test for elderly women. Physiother Theory Pract 2003, 19:35-43.

67. Hagstromer M, Troiano RP, Sjostrom M, Berrigan D: Levels and patterns of objectively assessed physical activity-a comparison between Sweden and the United States. Am J Epidemiol 2010, 171:1055-1064.

68. Fahn SER: Unified Parkinson's disease rating scale. In Recent Developments in Parkinson Diseases. Edited by Fahn SMD, Calne D. London: Macmillan; 1987:153-163.

69. Yardley L, Beyer N, Hauer K, Kempen G, Piot-Ziegler C, Todd C: Development and initial validation of the Falls Efficacy ScaleInternational (FES-I). Age Ageing 2005, 34:614-9.

70. Brown CA, Cheng EM, Hays RD, Vassar SD, Vickrey BG: SF-36 includes less Parkinson Disease (PD)-targeted content but is more responsive to change than two PD-targeted health-related quality of life measures. Qual Life Res 2009, 18:1219-1237. 
71. Sullivan M, Karlsson J, Ware JE Jr: The Swedish SF-36 Health Survey-I. Evaluation of data quality, scaling assumptions, reliability and construct validity across general populations in Sweden. Soc Sci Med 1995, 41:1349-58.

72. Fitzpatrick R, Peto V, Jenkinson C, Greenhall R, Hyman N: Health-related quality of life in Parkinson's disease: a study of outpatient clinic attenders. Mov Disord 1997, 12:916-922.

73. Duncan RP, Earhart GM: Randomized controlled trial of community-based dancing to modify disease progression in Parkinson disease. Neurorehabil Neural Repair 2012, 26:132-143.

74. Yogev-Seligmann G, Giladi N, Brozgol M, Hausdorff JM: A training program to improve gait while dual tasking in patients with Parkinson's disease: a pilot study. Arch Phys Med Rehabil 2012, 93:176-181.

75. Morris ME, Menz HB, McGinley JL, Huxham FE, Murphy AT, lansek R, Danoudis M, Soh SE, Kelly D, Watts JJ: Falls and mobility in Parkinson's disease: protocol for a randomised controlled clinical trial. BMC Neurol 2011, 11:93.

76. Canning CG, Sherrington C, Lord SR, Fung VS, Close JC, Latt MD, Howard K, Allen NE, O'Rourke SD, Murray SM: Exercise therapy for prevention of falls in people with Parkinson's disease: a protocol for a randomised controlled trial and economic evaluation. BMC Neurol 2009, 9:4.

77. Tomlinson CL, Stowe R, Patel S, Rick C, Gray R, Clarke CE: Systematic review of levodopa dose equivalency reporting in Parkinson's disease. Mov Disord 2010, 25:2649-2653.

doi:10.1186/1471-2377-12-111

Cite this article as: Conradsson et al: A novel conceptual framework for balance training in Parkinson's disease-study protocol for a randomised controlled trial. BMC Neurology 2012 12:111.

\section{Submit your next manuscript to BioMed Central and take full advantage of:}

- Convenient online submission

- Thorough peer review

- No space constraints or color figure charges

- Immediate publication on acceptance

- Inclusion in PubMed, CAS, Scopus and Google Scholar

- Research which is freely available for redistribution 